\title{
Analysis of stability to cheaters in models of antibiotic degrading microbial communities
}

\author{
András Szilágyi ${ }^{\mathrm{a}, \mathrm{b}}$, Gergely Boza ${ }^{\mathrm{c}, \mathrm{d}}$, István Scheuring ${ }^{\mathrm{a}, *}$ \\ ${ }^{a}$ MTA-ELTE, Theoretical Biology and Evolutionary Ecology Research Group Department of \\ Plant Systematics, Ecology and Theoretical Biology, Pázmány Péter sétány 1/c, Budapest, \\ 1117, Hungary \\ ${ }^{b}$ MTA Centre for Ecological Research, Evolutionary Systems Research Group, Klebelsberg \\ K. u. 3, Tihany, 8237, Hungary \\ ${ }^{c}$ Eötvös University, Department of Plant Systematics, Ecology and Theoretical Biology, \\ Pázmány Péter sétány 1/c, Budapest, 1117, Hungary \\ ${ }^{d}$ International Institute for Applied Systems Analysis (IIASA), Evolution and Ecology \\ Program and Risk and Resilience Program, Schlossplatz 1, Laxenburg, A-2361, Austria
}

\begin{abstract}
Antibiotic resistance carried out by antibiotic degradation has been suggested recently as a new mechanism to maintain coexistence of microbial species competing on a single limiting resource, even in well-mixed homogeneous environments. Species diversity and community stability, however, critically depend on resistance against social cheaters, mutants that do not invest in production, but still enjoy the benefits provided by others. Here we investigate how different mutant cheaters affect the stability of antibiotic producing and degrading microbial communities. We consider two cheater types, production and degradation cheaters. We generalize the mixed inhibition-zone and chemostat models introduced previously (Kelsic et al., 2015) to study the population dynamics of microbial communities in well-mixed environment, and analyze the invasion of different cheaters in these models. We show that production cheaters, mutants that cease producing antibiotics, always destroy coexistence whenever there is a cost of producing these antibiotics. Degradation cheaters, mutants that loose their function of producing extracellular antibiotic degrading molecules, induce community collapse only if the cost of producing the degradation factors is above
\end{abstract}

\footnotetext{
* Corresponding author

Email address: istvan.scheuring@ttk.elte.hu (István Scheuring)
} 
a critical level. Our analytical studies, supported by numerical simulations, highlight the sensitivity of antibiotic producing and degrading communities to loss-of-function mutants.

Keywords: rock-paper-scissors, social parasite, evolutionary instability, antibiotic-mediated microbiome, degradation resistance

\section{Introduction}

2

Unraveling mechanisms that maintain high genetic and functional diversity of microbial communities has become one of the most challenging problems in theoretical and evolutionary ecology (Costello et al., 2012; Morris et al., 2012; Cordero and Polz, 2014). A great variety of bacteria form stable communities in relatively homogeneous environments, competing for only a few limiting resources (Hibbing et al., 2010), seemingly contradicting with the competitive exclusion principle, which states that the number of species cannot be higher than the number of limiting resources (Gause, 1934).

In bacteria, the most common forms of interactions are carried out by molecules secreted into the extracellular environment, such as exoenzymes to digest nutrients (Arnosti, 2011), iron scavenging siderophores (Ross-Gillespie et al., 2009), signaling molecules (Miller and Bassler, 2001), virulence factors (Hacker and Carniel, 2001), antibiotics (Bernier and Surette, 2013), or antibiotic degrading molecules (Wright, 2005). Via these molecules, microorganisms can be in competitive, antagonistic, or cooperative relationships (West et al., 2001; Coyte et al., 2015). Interestingly, these molecules are public goods, meaning that not only the producers, but all nearby individuals can enjoy the benefits delivered by them (West et al., 2001). Cheaters, individuals that do not produce such molecules and hence pay no cost of production, can also enjoy these benefits. Thus cheaters have higher fitness and can outcompete producers, leading to the loss of the diversity by ceasing the production of the public good (West et al., 2001). These antagonistic interactions carried out by the extracellular antibiotics make cyclic competition dominance possible, for example, among 
Figure 1: Cyclical competition dominance of three species. (a) Topology of a general 'rockpaper-scissors' type interaction. Here species 1 wins over species 2 , species 2 wins over species 3 , and species 3 wins over species 1 , as indicated by the arrows. (b) The interaction topology where each species inhibits another by producing antibiotic (solid lines) and decomposes antibiotic produced by that species (dotted lines) according to a cyclical interaction topology. (Kerr et al., 2002; Károlyi et al., 2005).

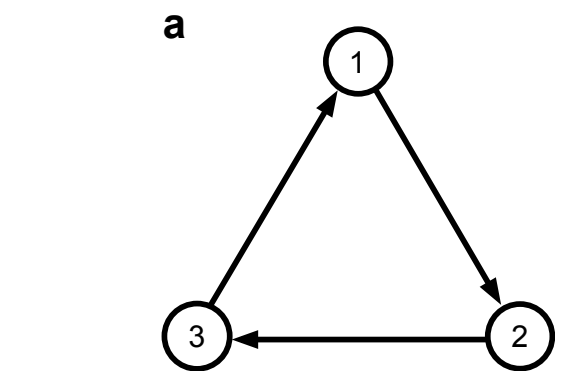

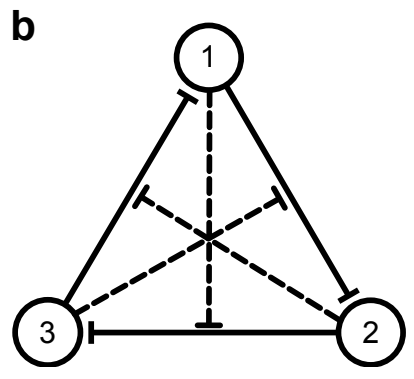

antibiotic sensitive, producer, and resistant types. Since producing of an antibiotic and being resistant to it are both costly, the resistant strain wins over the producer, similarly the sensitive wins over the resistant, and the producer can take over the sensitive population. This 'rock-paper-scissors' interaction cycle is the simplest example of cyclical competition dominance network, where each species is superior to one, but inferior to another (Fig. 1.a). Coexistence of species in such cyclical interaction networks is documented in spatially structured environments, in which interaction and dispersion are limited to the immediate neighbors of the focal individual (Kerr et al., 2002; Czárán et al., 2002; Károlyi et al., 2005; Müller and Gallas, 2010), but coexistence is much less prevalent in unstructured environments where individuals mix intensively

Recently, Kelsic et al. (2015) (KEA) employed theoretical models to demonstrate that bacterial species with different antibiotic production, intrinsic resistance, and extracellular degradation factors can coexist even in well-mixed microbial communities competing for one common limiting factor. Including degradation resistance has a key role in their model, since excreting antibiotic degrading molecules can weaken the inhibitory interaction between other species thus balance the fitnesses through the community. Their study focuses mainly 
on three species systems, in which species produce one type of antibiotics and reduce the effect of another type via degrading molecules (Fig. 1.b). The authors showed that coexistence of species in this system is robust to variation of model parameters even in well-mixed environment. They further demonstrated that analogous systems with four or five species producing 4-6 different antibiotics and degradation factors can have coexistence, although robustness is significantly less prevalent in these richer communities (Kelsic et al., 2015). However, the explanatory power and significance of degradation resistance in explaining microbial diversity largely depends on whether these communities prove to be resistant to the invasion of mutants, mainly against the invasion of social cheaters. A community is defined to be resistant or robust to the invasion of a mutant if its species composition does not change significantly after the invasion. That is, the mutant will be present in the community only transiently, and after its disappearance, the community returns to its pre-invasion state.

In the following, we study the generalized versions of KEA's so-called mixed inhibition-zone and chemostat models (Kelsic et al., 2015), and show analytically that bacterial communities, independently of the interaction topology, are not robust against the invasion of social cheaters. More precisely, we show that mutant cheaters, loosing the costly function of antibiotic production, destroy any diverse community either in one step, or following a cascade of invasion steps. The other type of social cheaters considered in the model, the mutants loosing their functions of producing extracellular antibiotic degrading molecules have less dramatic effect on community stability, but species diversity still declines after the invasion of such mutants.

\section{Model description}

We assume that there are $n_{s}$ phenotypically different species and $n_{a}$ different antibiotics that can be produced by these species. A phenotype (or species) is defined by its relation to an antibiotic: it can produce, can be resistant to, or can be sensitive to the given antibiotic. Naturally, a species producing an antibiotic 
is also resistant to it, where the resistance is carried out either by removing antibiotic molecules from the cell via efflux mechanisms, or by neutralizing these molecules within the cell (Kumar and Schweizer, 2005). Accordingly, a cell producing an antibiotic $l\left(P_{l}\right)$ is also intrinsically resistant $\left(R_{l}\right)$ to this antibiotic. Non-producing species can have two types of resistance: intrinsic resistance $\left(R_{l}\right)$ and degradation resistance $\left(D_{l}\right)$. Bacteria with degradation resistance produce molecules and secrete to the extracellular matrix which diffuse and degrade the target antibiotic molecules in a given neighborhood of the cell (Wright, 2005; Bastos et al., 2015). Phenotypes which are not resistant to antibiotics $l$ carried out either by intrinsic or by degradation resistance, are considered sensitive $\left(S_{l}\right)$ and the presence of this antibiotic in the locality reduces their fitnesses. Thus, every species $i=1,2, . . n_{s}$ is characterized by any of the four phenotypes $P_{l}, R_{l}, D_{l}, S_{l}$ for each antibiotic $l=1,2, . . n_{a}$.

Let $x_{i}$ be the abundance of species $i$ per unit area, and assume that cells are dispersed randomly on a two-dimensional surface. The fitness $w_{i}$ of species $i$ is determined by its intrinsic replication rate $g_{i}$ and the fraction of area $1-A_{i}^{(k i l l)}$ in which individuals of species $i$ are not killed by antibiotics, that is

$$
w_{i}=g_{i}\left(1-A_{i}^{(k i l l)}\right)
$$

Antibiotic $l$ is effective within area $K_{l}^{(P)}$ around the cell producing it and, similarly, degrading molecules protect every sensitive cell within area $K_{l}^{(D)}$ around a cell producing this degrading molecule. A sensitive cell is killed if there is at least one cell producing antibiotic $l$ within its $K_{l}^{(P)}$ neighborhood and there is no bacterium producing degrading molecules for antibiotic $l$ within its $K_{l}^{(D)}$ neighborhood. Since the aim of this model is to show that coexistence is possible in unstructured environment, it is assumed that bacteria are dispersed randomly, so the number of cells follows Poisson distribution within the defined areas. Thus, the probability that at least one antibiotic producer cell is in the $K_{l}^{(P)}$ neighborhood of a cell is $1-e^{-K_{l}^{(P)} x_{p}}$, where $x_{p}$ is the abundance of species producing antibiotic $l$. This value gives the fraction of area in which sensitive cells are killed except if they are protected by individuals producing degrading 
molecules within area $K_{l}^{(D)}$. If the abundance of species producing degrading molecules is $x_{d}$, then the probability of having no cells in this area is $e^{-K_{l}^{(D)} x_{d}}$. So, species $i$ is killed by antibiotic $l$ in the fraction of area is as follows

$$
A_{i, l}\left(x_{d}, x_{p}\right)=e^{-K_{l}^{(D)} x_{d}}\left(1-e^{-K_{l}^{(P)} x_{p}}\right) .
$$

Since not only one species can produce antibiotics $l$ or molecules degrading it, the total area where at least one molecule of antibiotic $l$ kills the sensitive species $i$ is written as a product of the probabilities of all possible occurrences

$A_{i, l}\left(x_{1}, x_{2} \ldots x_{i-1}, x_{i+1} \ldots x_{n_{s}}\right)=A_{i, l}\left(\mathbf{x} \backslash x_{i}\right)=\prod_{j=1}^{n_{s}} e^{-\delta_{j l} K_{l}^{(D)} x_{j}}\left(1-\prod_{j=1}^{n_{s}} e^{-\epsilon_{i j l} K_{l}^{(P)} x_{j}}\right)$,

where $\delta_{j l}=1$ if the $j$-th species degrades antibiotic $l$, otherwise $\delta_{j l}=0$. Similarly, $\epsilon_{i j l}=1$ if species $i$ is sensitive to antibiotic $l$ which is produced by species $j$, otherwise $\epsilon_{i j l}=0$ (for $P$ and $D$ type cells). Consequently, the fraction of area where individuals of species $i$ are not killed by any antibiotics of any other species is

$$
1-A_{i}^{(k i l l)}\left(\mathbf{x} \backslash x_{i}\right)=\prod_{l=1}^{n_{a}}\left(1-A_{i, l}\left(\mathbf{x} \backslash x_{i}\right)\right) .
$$

Thus, the fitness of species $i$ will be

$$
w_{i}=g_{i}\left(1-A_{i}^{(k i l l)}\left(\mathbf{x} \backslash x_{i}\right)\right),
$$

and the average fitness is

$$
\bar{w}=\sum_{i=1}^{n_{s}} w_{i} x_{i} .
$$

By knowing fitness functions for every species, the population dynamics of the system can be described by the following discrete-time replication dynamics:

$$
x_{i}(t+1)=\frac{c+w_{i}(t)}{c+\bar{w}(t)} x_{i}(t),
$$

where the $c>0$ constant depends on the time unit (Weibull, 1997). For the continuous time counterpart of the dynamics, see Appendix A.

We note here that KEA have pointed out previously, that the three-species coexistence (see Fig 1.b) is robust if the areas of chemical activities $\left(K_{l}^{(P)}\right.$ and 
$\left.K_{l}^{(D)}\right)$ and replication rates $\left(g_{i}\right)$ of all the three species are relatively similar. KEA have also shown that the same dynamics can be observed in the agentbased and the chemostat versions of the mixed inhibition-zone model (Kelsic et al., 2015). The detailed analyses of the generalized chemostat model can be found in Appendix C. They studied a system where $K_{l}^{(P)}=K^{(P)}$ and $K_{l}^{(D)}=$ $K^{(D)}$ are constants for every antibiotic which assumption does not have to hold in our generalized model.

Besides the ecological stability of three species models, KEA investigated the invasion of "production cheaters", that is, the mutants which do not produce antibiotics and "degradation cheaters" which do not produce degrading molecules. Losing these functions results in fitness increase for mutants, which is then translated into higher replication rates. Based on numerical simulations including cheaters in the community, they concluded that "These interactions enable coexistence that is robust to substantial differences in inherent growth rates and to invasion by 'cheating' species that cease to produce or degrade antibiotics." Our discussions with the authors clarified that they studied the evolutionary stability of this system in the spatially extended agent-based version of the mixed inhibition zone model, and analyzed it numerically for 3- and 4-species networks (Kelsic et al., 2015, 2016). They found that networks are resistant to both degradation and production parasites in these systems if the colonization radius is small enough. In the following sections, we show that cheater mutants crash such communities not only in the three-species 'rock-paper-scissors' interaction topology in the mixed inhibition model, but in the generalized mixed inhibition model, and similarly in the chemostat model with any interaction topology. In the discussion we explain briefly why the agent-based model with short range colonization behaves differently from the analytical model studied here. 


\section{Results}

3.1. Evolutionary instability in the mixed inhibition-zone model: introducing social cheaters

Species having resistance $D_{l}$ protect not only themselves but any other strains $S_{l}$ in the neighborhood from the antibiotics, and similarly a strain $P_{l}$ producing antibiotic $l$ generates empty space by killing sensitive individuals not only for itself but for non-producing strains $R_{l}$ as well. Therefore these degrading molecules and antibiotics are public goods, so strains not producing the costly degradation or antibiotic molecules have advantage over producers; thus these are social cheaters (Hardin, 1968; Cordero et al., 2012b). We consider two types of mutants, "production cheaters" that fail to produce antibiotics but retain intrinsic resistance to this antibiotic $\left(P_{l} \rightarrow R_{l}\right)$, and "degradation cheaters" that lose their resistance through antibiotic degradation and become susceptible to the antibiotics $\left(D_{l} \rightarrow S_{l}\right)$. The benefit of non-producing extracellular materials results in higher replication rates for cheaters, that is the growth rate of mutant increases with $(1+\alpha)$, where $\alpha$ is an arbitrary, but generally small, positive number.

\subsubsection{Invasion of antibiotic production cheaters}

Assume that an antibiotic production cheater evolves in a community in which $n_{s}$ species are in a stable coexistence. (According to KEA, any type of species coexistence is possible from stable fixed points through limit cycles to chaotic behaviors. Our analysis remains valid for every type of dynamical coexistence.) Let us denote the mother species by $m$, and assume this species produces antibiotic $l$. The mutant $m^{\prime}$ of the mother looses the costly production of antibiotic $l$ and consequently its replication rate increases as $g_{m^{\prime}}=g_{m}(1+\alpha)$. It follows from the definition of the model that the fitness function of species $m$ depends only on the abundances of the two types of species affecting survival: the species producing antibiotics for which the focal species is sensitive, and the species producing the molecules degrading this particular antibiotic (see 
Eq. 3). Since $m^{\prime}$ remains sensitive to the same antibiotic as $m$, its replication rate increases, but its fitness function does not change. Thus, the dynamics of mother and mutant species are

$$
\begin{aligned}
x_{m}(t+1) & =\frac{c+w_{m}(t)}{c+\bar{w}^{\prime}(t)} x_{m}(t) \\
x_{m^{\prime}}(t+1) & =\frac{c+w_{m^{\prime}}(t)}{c+\bar{w}^{\prime}(t)} x_{m^{\prime}}(t),
\end{aligned}
$$

where $\bar{w}^{\prime}(t)$ is the average fitness in the population including the mutant. Dividing Eq. (8) by Eq. (9)

$$
\frac{x_{m}(t+1)}{x_{m^{\prime}}(t+1)}=\frac{c+w_{m}}{c+(1+\alpha) w_{m}} \frac{x_{m}(t)}{x_{m^{\prime}}(t)}
$$

that is

$$
\frac{x_{m}(t+1)}{x_{m^{\prime}}(t+1)}=\left[\frac{c+w_{m}(t)}{c+(1+\alpha) w_{m}(t)}\right]^{t} \frac{x_{m}(0)}{x_{m^{\prime}}(0)} .
$$

Since $0<\left[c+w_{m}(t)\right] /\left[c+(1+\alpha) w_{m}(t)\right]<1$ for any $c \geq 0$ then

$\lim _{t \rightarrow \infty}\left(\left[c+w_{m}(t)\right] /\left[c+(1+\alpha) w_{m}(t)\right]\right)^{t}=0$ and consequently

$$
\lim _{t \rightarrow \infty} x_{m}(t) / x_{m^{\prime}}(t)=0
$$

According to (12) three scenarios are possible: (i) both $m$ and $m^{\prime}$ are selected against in the community, but species $m$ goes extinct faster than species $m^{\prime}$; (ii) species $m$ is selected against, and the invading mutant $m^{\prime}$ is getting fixed in the community, but mutant $m^{\prime}$ triggers the loss of another species besides the mother strain; (iii) species $m$ is selected against, and species $m^{\prime}$ replaces it in the community, so the number of coexisting species remains unchanged. In case of scenarios (i) and (ii), the number of coexisting species decreases after the invasion of the mutant. In scenario (iii) a non-producing cheater merely replaces a producer.

Let us assume a sequence of production cheaters invading according to (iii). The number of coexisting species doesn't change in this scenario, however if there were $n_{a}$ number of different antibiotics in the community then the number of antibiotics decreases to zero after at most $n_{a}$ number of such a species replacements. As a result, neither of the coexisting species produces antibiotics any more in this new community. However, survival of more than one 
species becomes impossible in this situation, since the replication rate will become $w_{i}=g_{i}$ for every $i$ as there are no more interactions between the species, and thus only the species with the highest $g_{i}$ will survive (survival of the fittest). Consequently, in any of the above mentioned possible scenarios, species $m$ (and consequently the community) is not resistant against the invasion of mutant $m^{\prime}$ that has any replication benefit $(\alpha>0)$ due to its loss of antibiotic producing function. We show that continuous time replicator dynamics and the chemostat model lead to completely similar results (see Appendix A and C for details).

\subsubsection{Invasion of degradation cheaters}

The other type of social cheater is the degradation cheater $m^{\prime}$, which ceases the production of degradation molecule synthesized by the mother species $m$ against antibiotic $l$. By loosing this function, $m^{\prime}$ becomes sensitive to antibiotic $l$ if it is present in the environment but its replication rate increases as $g_{m}(1+\alpha)$ at the same time. Thus, the equations of the mother and the mutant species dynamics are

$$
\begin{aligned}
x_{m}(t+1) & =\frac{c+w_{m}(t)}{c+\bar{w}^{\prime}(t)} x_{m}(t) \\
x_{m^{\prime}}(t+1) & =\frac{c+(1+\alpha)\left(1-A_{m^{\prime}, l}\left(\mathbf{x} \backslash x_{m^{\prime}}\right)\right) w_{m}(t)}{c+\bar{w}^{\prime}(t)} x_{m^{\prime}}(t) .
\end{aligned}
$$

Dividing Eq. (13) by Eq. (14) we get

$$
\frac{x_{m}(t+1)}{x_{m^{\prime}}(t+1)}=\left[\frac{c+w_{m}(t)}{c+(1+\alpha)\left(1-A_{m^{\prime}, l}\left(\mathbf{x} \backslash x_{m^{\prime}}\right)\right) w_{m}(t)}\right]^{t} \frac{x_{m}(0)}{x_{m^{\prime}}(0)}
$$

The fate of a mutant depends on the values of both $\alpha$ and $A_{m^{\prime}, l}\left(\mathbf{x} \backslash x_{m^{\prime}}\right)$, thus the advantage of the invading mutant $m^{\prime}$ is insufficient yet. By defining $A_{m^{\prime}, l}^{(\max )}=\operatorname{Max}\left\{A_{m^{\prime}, l}\left(\mathbf{x} \backslash x_{m^{\prime}}\right) \mid x_{i} \in[0,1], \sum_{i} x_{i}=1\right\}$ a sufficient condition for the invasion of mutant $m^{\prime}$ can be set. For $\lim _{t \rightarrow \infty} x_{m}(t) / x_{m^{\prime}}(t)=0$ to be valid, the expression in the square bracket on the right hand side of (15) must be in the $(0,1)$ interval which leads to the following sufficient condition:

$$
\alpha>\frac{A_{m^{\prime}, l}^{(\max )}}{1-A_{m^{\prime}, l}^{(\max )}} .
$$


Consequently, one of the above mentioned three possible scenarios describes the fate of mutant $m^{\prime}$ in this case as well. However, besides the loss of species diversity, according to the above described three invasion scenarios, it is possible that the degradation-molecule producer and the sensitive mutant strains coexist. To prove this we show that it is possible that $m^{\prime}$ invades the community where type $m$ is resident, but $m$ invades the community where $m^{\prime}$ is resident. Let us assume first that $m$ is resident in a stably coexisting community. For the sake of simplicity, we assume that coexistence is characterized by a stable fixed point, denoted by $\hat{\mathbf{x}}^{(\mathbf{1})}$. The mutant $m^{\prime}$ emerges in small abundance, that is $x_{m}^{\prime} \ll \hat{x}_{i}^{(1)}$ for every $i \neq m^{\prime}, \hat{x}_{i}^{(1)}>0$. Since $x_{i}(t+1)=x_{i}(t)$ for every $i, \hat{x}_{i}^{(1)}>0$ at the equilibrium the abundance of the rare mutant $m^{\prime}$ increases in the community if (cf. Eq. (14))

$$
\frac{c+(1+\alpha)\left(1-A_{m^{\prime}, l}\left(\hat{\mathbf{x}}^{(\mathbf{1})} \backslash x_{m^{\prime}}\right)\right) w_{m}(t)}{c+\bar{w}^{\prime}(t)}>1,
$$

which leads to the condition

$$
\alpha>\frac{A_{m^{\prime}, l}\left(\hat{\mathbf{x}}^{(\mathbf{1})} \backslash x_{m^{\prime}}\right)}{1-A_{m^{\prime}, l}\left(\hat{\mathbf{x}}^{(\mathbf{1})} \backslash x_{m^{\prime}}\right)} .
$$

Let us consider now $m^{\prime}$ as the resident species of the same community but $m$ is replaced by $m^{\prime}$ and thus $m$ is the rare mutant. Let $\hat{\mathbf{x}}^{(\mathbf{2})}$ denote the equilibrium abundances before invasion, so the rare mutant $m$ spreads if

$$
\frac{c+\frac{w_{m^{\prime}}(t)}{(1+\alpha)\left(1-A_{m^{\prime}, l}\left(\hat{\mathbf{x}}^{(2)} \backslash x_{m^{\prime}}\right)\right)}}{c+\bar{w}^{\prime}(t)}>1,
$$

(cf. Eq. (14) that is if

$$
\alpha<\frac{A_{m^{\prime}, l}\left(\hat{\mathbf{x}}^{(\mathbf{2})} \backslash x_{m^{\prime}}\right)}{1-A_{m^{\prime}, l}\left(\hat{\mathbf{x}}^{(\mathbf{2})} \backslash x_{m^{\prime}}\right)} .
$$

Consequently, if $A_{m^{\prime}, l}\left(\hat{\mathbf{x}}^{(\mathbf{2})} \backslash x_{m^{\prime}}\right)<A_{m^{\prime}, l}\left(\hat{\mathbf{x}}^{(\mathbf{1})} \backslash x_{m^{\prime}}\right)$ then both (18) and (20) can be satisfied simultaneously, thus the rare $m$ and $m^{\prime}$ mutants mutually invade the communities in which the other is resident, which guarantees the coexistence of these species. Naturally, this analysis assumes that beside species $m$ and $m^{\prime}$ there is at least one another species that produces an antibiotic lethal 
for species $m^{\prime}$. Furthermore, it is assumed that residents $m$ and $m^{\prime}$ are in coexistence with the same species, but their densities can be different. Identical conditions determine the invasion of mutants in a model based on continuous replicator dynamics (see Appendix B for details). Thus, according to our analytical investigation, degradation cheaters can coexist within the resident community, and can degrade resident community only if their replication rate is above a critical level. This level can be arbitrarily low or high depending on the parameters. In the next section, we will test the generality of our results using numerical investigations.

\subsection{Numerical studies}

Next, we run numerical investigations to test the effect of social cheaters, and for comparison we followed the methodology and parameters used by KEA in their simulations. In the first series of experiments we generated a statistically representative sample of ecologically stable communities of 3-5 coexisting species producing 2-5 different antibiotics, where the initially selected five species can be any of the four phenotypes $\left(S_{l}, D_{l}, R_{l}, P_{l}\right)$ for each antibiotic $l=1,2, \ldots, 5$ and the intrinsic replication rate for species $i$ is: $g_{i}=1+(i-1) \cdot 0.005$. The area of chemical activities were either $K_{l}^{(P)}=K^{(P)}=10$ and $K_{l}^{(D)}=K^{(D)}=3$ or $K_{l}^{(P)}=K^{(P)}=30$ and $K_{l}^{(D)}=K^{(D)}=10$. We randomly assembled communities with five interacting species by assigning randomly selected phenotypes for each antibiotic $l$ to each of the species. The initial abundances were $1 / n_{s}$ for each species. We repeated $T=10.000$ update steps according to Eq. (7) with $c=0$ and determined the number of coexisting species and the type of equilibrium at the end (fixed point, limit cycle or chaotic behavior). (We note that $c=0$ is the standard parameter choice used by KEA as well, although $c>0$ fits the mathematical deduction of the dynamics (Weibull, 1997). However, this modification does not alter the qualitative behavior of the model.) A species was considered to be extinct if its frequency went below $0.01 / n_{s}$ (Kelsic et al., 2015).

In agreement with Kelsic et al. (2015, Extended data Figure 8), we experi- 
enced that only an extremely small fraction of possible interaction topologies were suitable to maintain complex communities. While three species remain in coexistence from the the initial five species networks in 1 out of $10^{2}-10^{3}$ randomly selected networks, five species could coexist only in 1 out of $10^{4}-10^{6}$ randomly selected networks on average (depending on the $K^{(P)}$ and $K^{(D)}$ parameters). That is, in line with the Extended Data Figure 8 of Kelsic et al. (2015), we found that the fraction of stable communities decreases dramatically as the number of coexisting species increases.

After generating the sample of ecologically stable 3-5 species communities we tested the resistance of these communities against the production and degradation cheaters but only one function and only in one species could be lost at a time, thus either $\mathrm{P} \rightarrow \mathrm{R}$ or $\mathrm{D} \rightarrow \mathrm{S}$ mutants could emerge in the community for each possible case. The mutants with fitness of $(1+\alpha) g_{i}$ were introduced at the 10.000th time step with density of $10^{-3}$, and the density of the corresponding mother species was decreased by the same amount. After subsequent 10.000 update steps the coexistence was monitored again, and we recorded the communities that could not resist invasion and hence diversity declined. We declared communities not being resistant to the invasion of mutants if at least one mutant type caused the number of coexisting species (with frequency higher than 0.01) to be smaller after $T$ time steps compared to the number of species before the invasion. That is, we consider only the cases when the invasion of mutants decreases the number of coexisting species within one step (scenarios (i) and (ii)).

We tested the resistance of three, four, and five-species communities against the cheater mutants as the function of the $\alpha$ growth-rate advantage of the mutants. There is a critical $\alpha$ above which the fraction of unstable communities increases abruptly in a sigmoid manner (Fig. 2a). Species diversity declines dramatically in the majority of these communities even at as little as $0.1 \%$ relative growth-rate advantage of mutants $\alpha^{*}=\alpha / \bar{g}_{i}$ where $\bar{g}_{i}$ is the average growth rate in the community. The rapid decline of diversity results in the exclusion of all but one species in most of the cases (around $70 \%$ of the outcomes in the 

for the decline of diversity in more than $99 \%$ of the cases.
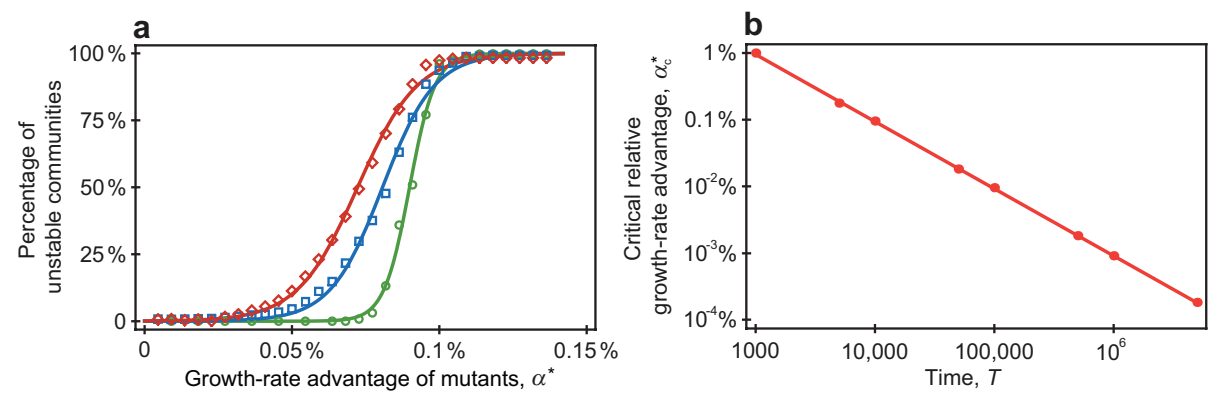

Figure 2: Measures of community instability fostered by cheater mutants. (a) The fraction of unstable communities increases in a sigmoid manner (depicted by colored lines) as the relative growth-rate advantage of cheater mutants increases. At $0.1 \%$ growth-rate advantage, the majority of the modeled communities become unstable. Statistics are based on $10^{3}$ randomly selected communities composed of three (green circles), four (blue rectangles), and five (red diamonds) species. (b) The critical level of relative growth-rate advantage of mutants (where at least $99 \%$ of communities are not resistant to the invasion of at least one mutant type) decreases as the duration of simulations $(T)$ increases for $10^{3}$ randomly selected interaction network topologies composed of 5 species. Parameters are: $g_{i}=1+(i-1) \cdot 0.05, K_{j}^{(P)}=$ $K^{(P)}=30, K_{j}^{(D)}=K^{(D)}=10$.

In our second analysis, we studied the dependence of community resistance on simulation time. According to Eq. (11), it is straightforward to assume that it takes more time to observe competitive exclusion if fitness differences are smaller. To test this hypothesis, we repeated the numerical experiments in five-species communities with parameters used in Figure 2a but for different simulation times $(T)$, and measured the critical $\alpha_{c}^{*}$, that is the $\alpha^{*}$ value for which at least $99 \%$ of the communities proved to be unstable. As Figure $2 \mathrm{~b}$ demonstrates, $\alpha_{c}^{*}$ decreases continuously as the duration of the simulations increases according to $\alpha_{c}^{*} \propto T^{-1.05 \pm 0.01}$. This relation is in concordance with our analytical results, since the necessary condition to detect collapse of community is that $x_{m}(t) / x_{m^{\prime}}(t) \leq x_{c}$ where $x_{c}$ is a critical frequency below which 
the species is selected out by definition. It follows from Eq. (11) that

$$
\ln \left(x_{c}\right)=T \ln \left(\frac{1}{1+\alpha}\right) .
$$

For $\alpha \ll 1 \ln [1 /(1+\alpha)] \approx-\alpha$, consequently $\alpha \propto 1 / T$ determines the relationship between these two variables in the extinction dynamics.

To investigate the different invasion scenarios discussed previously, we numerically analyzed the invasion dynamics of different production and degradation cheaters in a community with the topology shown in Figure 3a. Note that in this case antibiotic production - sensitivity combinations are not cyclic as in Figure 1, but still each antibiotic is degraded by one of the species. This topology enables us to demonstrate all possible invasion events starting from the same community. We iterated the dynamics for 1000 time steps and then introduced mutants into the system. The number of coexisting species was monitored until $t=2000$ (except in Fig. 4d in which case due to slow invasion dynamics the mutant was added at $t=2000$ and the simulation was terminated at $t=4000$ ).

Investigating the three invasion scenarios in the numerical model discussed previously (see Eq. (12) and afterwards) confirms that the invasion of mutants can (i) result in the extinction of both the mutant and the mother species (Fig. 3b); (ii) result in the exclusion of mother species leading to a decrease in species diversity (Fig. 3c); and (iii) exclude the mother species but the mutant remains in coexistence with the other species (Fig. 3d).

Figure $3 \mathrm{~b}$ shows the effect of the invasion of production cheater mutant for species 2 (mutant ceases producing the antibiotic that inhibits species 5). Although the invasion of this mutant is unsuccessful it triggers a community collapse and only one resident species (species 5 in this case) remains in the end. In Figure 3c the other possible production cheater mutant of species 2 (mutant ceases producing the antibiotic that inhibits species 4) invades the system and reduces the number of coexisting species (to an odd number smaller than the original number of species; in our case to one).

Finally, in Figure 3d the same type of mutant with lower fitness advantage invades the community and replaces the mother species preserving the number of 
coexisting species but reducing the number of interactions by one. In accordance with Eq. (12) and discussions afterwards, these results suggest that the invasion of cheater mutants can result in the loss of species diversity, antibiotic diversity, or both.

In case of degradation cheater invasion experiments (in model community with the same topology as in Fig. 3a) we found the four different outcomes in line with expectations from Eq. (16) and the discussion afterwards. In contrast to production cheater mutants, degradation cheaters cannot always invade the system, thus the community structure can remain intact, or the mutants can coexist with the original coalition (Fig. 4). In line with the first scenario of the production mutants, the degradation cheater (mutant of species 5) can destroy the coexistence and one of the original species survives (Fig. 4c), or the cheater (mutant of species 2) survives only after the community collapses (Fig. 4d).

\section{Discussion}

Our results imply that the counteraction of antibiotic production by extracellular antibiotic degradation does not in itself guarantee high diversity in antibiotic producing microbial communities. In particular, we pointed out that production cheaters with increased reproduction rate demolish the coexistence of interacting species in well-mixed models. According to our studies, three scenarios are possible: in two cases (scenarios (i) and (ii)) the invasion of production cheaters causes immediate decrease of the number of coexisting species. In scenario (iii) it takes more than one invasion events to decrease the number of coexisting species, but eventually a sequence of invasion events also leads to the decrease of species diversity. These results are valid for the mixed inhibitionzone model and the chemostat model with any interaction topology and even if the different antibiotics and degradation molecules have different diffusion abilities (different $K_{l}^{(D)}$ and $K_{l}^{(P)}$ parameters). It follows that the invasion success of production cheaters is independent of the model details. Our conclusions remain valid for any other systems where the fitness of phenotype $i$ is 
described by $g_{i} f_{i}\left(x_{1}(t), x_{2}(t), x_{i-1}(t), x_{i+1}(t), ..\right)$, where $f_{i}\left(\mathbf{x} \backslash x_{i}\right)$ is an arbitrary continuous function and the replicator dynamic describes the selection among the different phenotypes (see Eqs. (9-12)). We found that the emergence of degradation cheaters causes less dramatic changes in the community; they are able to invade a stable community only if their fitness benefit is above a critical level, and in some cases the coexistence of mutant and resident types is possible after invasion.

Our numerical simulations show (in line with Kelsic et al. (2015) Extended Data Figure 8.) that the proportion of ecologically stable communities among randomly selected interaction topologies becomes negligibly low as the number of coexisting species increases to five or more. As in the current study the focus was on the evolutionary stability of microbial communities against invasion by cheaters, this aspect of ecological stability received less attention in our analyses. Similarly, in the study of KEA this behavior of the system did not receive sufficient attention. However, we would like to emphasize that it becomes increasingly unlikely that stable communities can emerge when the number of species increases. That is, besides the evolutionary instability, the robustness of ecological stability of these communities is also problematic in well-mixed models without additional mechanisms promoting diversity.

A more recent investigation by (Kelsic et al., 2016) pointed out that the spatially extended agent-based version of the mixed inhibition model exhibits resistance to invasion of cheaters. The crucial difference is that in this spatial extended model empty sites are colonized from a finite distance. A producer cell creates empty sites by killing sensitive cells in its neighborhood. Such cells have a greater chance for colonizing these empty sites than the non-producing cheaters being in the vicinity of the empty site. Thus producer cells have higher replication success than non-producers which can balance the higher per-capita replication rate of non-producer ones. The smaller the colonization distance the higher the benefit of producers compared to non-producers, and since the colonization distance tends to be infinite in the well-mixed models studied here this effect disappears. 
We assumed in the analysis that the production of antibiotics and molecules degrading antibiotics is costly for the cells. In line with this assumption, there are numerous experiments demonstrating that the inactivation or loss of such genes have a significant positive effect on the fitness of such mutant types in a given environment (Lee and Marx, 2012; Koskiniemi et al., 2012; D'Souza et al., 2014). Moreover, other investigations reveal that such antibiotic resistance factors can be the by-products of the general metabolism and thus the production costs are practically negligible (Melnyk et al., 2014). In some cases, switching off such gene can even be beneficial for the cell due to pleiotropic effects of the regulating genes (Dandekar et al., 2012; Mitri and Foster, 2016). However, the high population size which is typical in bacterial communities enhances selection and thus it can dominate over genetic drift even for small fitness differences.

The mixed inhibition-zone and chemostat models consider the dynamics of well-mixed individuals producing diffusive antibiotics and degrading molecules. The assumptions behind these models enable us to handle the problem analytically, however, these assumptions oversimplify some aspects of the dynamics. First and foremost a more realistic diffusion dynamics and chemical interactions among the dispersed molecules and cells are not taken into account. It is known from other studies that even minor modifications in the dynamics describing diffusion of public goods molecules, interaction of these molecules with cells, the non-linear relation between the molecule concentration and the fitness, and even timing of death and birth events in population dynamics can have significant effect on selection between producers and non-producers (Borenstein et al., 2013; Scheuring, 2014; Archetti, 2014).

Recent studies pointed out that the secreted extracellular molecules are not completely mixing public goods, because due to the restricted motion of cells and of molecules in real bacterial communities, only the immediate neighborhood of the producer is able to enjoy the benefits (Morris, 2015). As the close neighbors of the producer are most probably the clones of the producer, non-producers further away from the source can benefit much less. According to the experiments, these definite spatial effects establish density-dependent and negative 
frequency-dependent selection which stabilizes the coexistence of the producers and social cheaters (Kerr et al., 2002; Cordero et al., 2012a; Drescher et al., 2014; Kümmerli et al., 2014; Morris, 2015). In addition, our results highlight that interactions of antibiotic production and attenuation are insufficient in effectively stabilizing bacterial communities in well-mixed environments. Presumably microscale spacial structure of the habitat, negative frequency-dependent selection, pleiotropy, auxotrophy, and top down control by phages play more significant role in maintaining microbiome diversity (Cordero and Polz, 2014; Morris et al., 2012, 2014; Morris, 2015; Koskiniemi et al., 2012; D'Souza et al., 2014; Velend, 2010; Ross-Gillespie et al., 2007, 2009; Dandekar et al., 2012; Mitri and Foster, 2016; Kelsic et al., 2016).

\section{Acknowledgements}

This work was supported by OTKA grant (No K100299) and by GINOP grant (2.3.2-15-2016-00057). We thank Roy Kishony, Eric Kelsic and Kalin Vestigian and anonymous referees for their valuable comments on earlier version of the manuscript.

\section{Appendix A. Continuous replicator dynamics: invasion of produc-} tion cheaters

The continuous replication dynamics of bacterial strains is generally written as

$$
\dot{x}_{i}(t)=\left(w_{i}(t)-\bar{w}(t)\right) x_{i}(t),
$$

where $w_{i}(t)$ and $\bar{w}(t)$ are the fitness values of individuals and the population average as defined in the main text. Let us denote the mother and production cheater mutant with $m$ and $m^{\prime}$, respectively. Thus, the dynamics of these two types are

$$
\begin{aligned}
\dot{x}_{m}(t) & =\left(w_{m}(t)-\bar{w}^{\prime}(t)\right) x_{m}(t) \\
\dot{x}_{m^{\prime}}(t) & =\left((1+\alpha) w_{m}(t)-\bar{w}^{\prime}(t)\right) x_{m^{\prime}}(t) .
\end{aligned}
$$


Dividing the two equations by $x_{m}(t)$ and $x_{m^{\prime}}(t)$, respectively, and subtracting Eq. (A.3) from Eq. (A.2), after some rearrangement we get

$$
\frac{\dot{x}_{m}(t)}{x_{m}(t)}-\frac{\dot{x}_{m^{\prime}}(t)}{x_{m^{\prime}}(t)}=-\alpha w_{m}(t),
$$

which leads to

$$
\frac{x_{m}(t)}{x_{m^{\prime}}(t)}=e^{-\alpha \int_{0}^{t} w_{m}(\tau) d \tau}
$$

Since $w_{m}(t)>w_{\text {min }}>0$, where $w_{\text {min }}$ is a constant, we have $\lim _{t \rightarrow \infty} \int_{0}^{t} w_{m}(\tau) d \tau=$ $\infty$. Therefore, equation (12), and consequently the three scenarios described in the main text remain valid in continuous time dynamical systems as well.

\section{Appendix B. Continuous replicator dynamics: invasion of degrada-} tion cheaters

In case of continuous replicator dynamics, the time evolution of $m$ and $m^{\prime}$ species is

$$
\begin{aligned}
\dot{x}_{m} & =\left(w_{m}(t)-\bar{w}(t)\right) x_{m} \\
\dot{x}_{m^{\prime}} & =\left((1+\alpha) w_{m}(t)\left(1-A_{m^{\prime}, l}\left(\mathbf{x} \backslash x_{m^{\prime}}\right)\right)-\bar{w}^{\prime}(t)\right) x_{m^{\prime}}
\end{aligned}
$$

where $m^{\prime}$ denotes the degradation cheater. Following the algebraic steps described in the previous subsection, we get

$$
\frac{\dot{x}_{m}(t)}{x_{m}(t)}-\frac{\dot{x}_{m^{\prime}}(t)}{x_{m^{\prime}}(t)}=\left[1-(1+\alpha)\left(1-A_{m^{\prime}, l}\left(\mathbf{x} \backslash x_{m^{\prime}}\right)\right] w_{m}(t) .\right.
$$

The sign of the right hand side of (B.3) depends on $\alpha$ and $A_{m^{\prime}, l}\left(\mathbf{x} \backslash x_{m^{\prime}}\right)$. As before, a sufficient condition for the invasion of mutant $m^{\prime}$ can be determined with the help of the maximum value of $A_{m^{\prime}, l}\left(\mathbf{x} \backslash x_{m^{\prime}}\right)$ : if $\left[1-(1+\alpha)\left(1-A_{m^{\prime}, l}^{(\max )}\right)\right]<$ 0 , that is if

$$
\alpha>\frac{A_{m^{\prime}, l}^{(\max )}}{1-A_{m^{\prime}, l}^{(\max )}} .
$$

To determine the criterion of mutual invasibility, let us assume first that type $m$ is the resident species and type $m^{\prime}$ invades the community. For sake of simplicity (as in the discrete model presented in the main text), we assume 
that the dynamics of the resident population is in fixed point, the abundances before invasion are denoted by $\mathbf{x}^{(\mathbf{1})}$. Mutant $m^{\prime}$ spreads if

$$
\dot{x}_{m^{\prime}}(t)=\left((1+\alpha)\left(1-A_{m^{\prime}, l}\left(\hat{\mathbf{x}}^{(\mathbf{1})} \backslash x_{m^{\prime}}\right)\right) w_{m}(t)-\bar{w}(t)\right) x_{m^{\prime}}(t)>0
$$

which leads to

$$
\alpha>\frac{A_{m^{\prime}, l}\left(\hat{\mathbf{x}}^{(\mathbf{1})} \backslash x_{m^{\prime}}\right)}{1-A_{m^{\prime}, l}\left(\hat{\mathbf{x}}^{(\mathbf{1})} \backslash x_{m^{\prime}}\right)} .
$$

Let us consider now $m^{\prime}$ as the resident species in a community and $m$ as the rare mutant. Let $\hat{\mathbf{x}}^{(\mathbf{2})}$ denote the equilibrium abundances before invasion, so the rare mutant $m$ spreads if

$$
\dot{x}_{m}(t)=\left(\frac{w_{m^{\prime}}(t)}{(1+\alpha)\left(1-A_{m^{\prime}, l}\left(\hat{\mathbf{x}}^{(\mathbf{2})} \backslash x_{m^{\prime}}\right)\right)}-\bar{w}^{\prime}(t)\right) x_{m}(t)>0
$$

which leads to the condition

$$
\alpha<\frac{A_{m^{\prime}, l}\left(\hat{\mathbf{x}}^{(\mathbf{2})} \backslash x_{m^{\prime}}\right)}{1-A_{m^{\prime}, l}\left(\hat{\mathbf{x}}^{(2)} \backslash x_{m^{\prime}}\right)} .
$$

Again, as in the discrete time dynamics, if $A_{m^{\prime}, l}\left(\hat{\mathbf{x}}^{(\mathbf{2})} \backslash x_{m^{\prime}}\right)<A_{m^{\prime}, l}\left(\hat{\mathbf{x}}^{(\mathbf{1})} \backslash x_{m^{\prime}}\right)$ then both (B.6) and (B.8) can be satisfied simultaneously, thus the rare $m$ and $m^{\prime}$ mutants mutually invade each other which guarantees the coexistence of these species. (Naturally, this analysis assumes that beside species $m$ and $m^{\prime}$ at least one similar a species is present in the community which produces antibiotic affecting species $m^{\prime}$.)

\section{Appendix C. Invasion of production cheaters in the chemostat model}

Here we review the chemostat model version of microbial community with interference competition. Following Kelsic et al. (2015), it is assumed that bacteria compete for a common limiting resource $z$ and there is a constant dilution $d$ from the chemostat. The dynamics of the resource is

$$
\dot{z}(t)=\left(z_{0}-z(t)\right) d-\frac{\sum_{i=1}^{n_{s}} w_{i}(t) x_{i}(t)}{\mu},
$$

where $z_{0} d$ is the constant inflow into the chemostat, $w_{i}(t)$ is the actual growth rate of species $i$ with concentration $x_{i}$ and $\mu$ is a conversion factor between 
resource and species concentration. The species concentrations change according to

$$
\dot{x}_{i}(t)=\left(w_{i}(t)-d\right) x_{i}(t)
$$

with

$$
w_{i}(t)=g_{i} \frac{z(t)}{k_{z}+z(t)} \prod_{j=1}^{n_{a}} e^{-\sigma_{i, j} K_{j}^{(P)} c_{j}(t)},
$$

that is the growth rate $w_{i}(t)$ is determined by the intrinsic growth rate $g_{i}$, the concentrations of the resource and the antibiotics $z(t)$ and $c_{j}(t)$, respectively. The effect of $z$ is saturated in line with the standard Michaelis-Menten kinetics with half saturation constant $k_{z}$ and the antibiotics cause exponential decay on total growth rate, $\sigma_{i, j}=1$ if species $i$ is sensitive to antibiotic $j$ otherwise $\sigma_{i, j}=$ 0 . The concentration of the antibiotics changes because of the production, the degradation, and the dilution of antibiotics, thus the dynamics can be written as

$$
\dot{c}_{j}(t)=\rho \sum_{i=1}^{n_{s}} \eta_{i, j} w_{i}(t) x_{i}(t)-K_{j}^{(D)} c_{j}(t) \sum_{i=1}^{n_{s}} \delta_{i, j} x_{i}(t)-d c_{j}(t),
$$

where $\rho$ is the amount of antibiotics produced by unit concentration of cells, $\eta_{i, j}=1$ if antibiotic $j$ produced by species $i$, otherwise $\eta_{i, j}=0$. Similarly $\delta_{i, j}=1$ if species $i$ produces degradation molecules for antibiotic $j$, otherwise $\delta_{i, j}=0$. It follows from (C.1) and (C.2) that

$$
\frac{\mathrm{d}}{\mathrm{d} t}\left(\sum_{i=1}^{n_{s}} \frac{x_{i}(t)}{\mu}+z(t)-z_{0}\right)=-d\left(\sum_{i=1}^{n_{s}} \frac{x_{i}(t)}{\mu}+z(t)-z_{0}\right),
$$

thus after a transient time

$$
z(t)=z_{0}-\sum_{i} \frac{x_{i}(t)}{\mu}
$$

Therefore (C.1) can be eliminated when we study the stationary solutions of the system by substituting (C.6) into (C.3) (Kelsic et al., 2015).

Let us assume that dynamics of a bacterial community is described by (C.1C.4), and a species $m$ is a member of a community $\left(\bar{x}_{m}>0\right.$ in the stationary state), and produces at least one type of antibiotic. The mutant $m^{\prime}$ species looses the production of this antibiotic, thus it has an increased growth rate 
$\left(g_{m^{\prime}}=(1+\alpha) g_{m}, \alpha>1\right)$ as above. Thus, the difference of relative growth rates of $m$ and $m^{\prime}$ species is

$$
\frac{\dot{x}_{m}(t)}{x_{m}(t)}-\frac{\dot{x}_{m^{\prime}}(t)}{x_{m^{\prime}}(t)}=w_{m}(t)-w_{m^{\prime}}(t)=-\alpha \frac{z(t)}{k_{z}+z(t)} \prod_{j=1}^{n_{a}} e^{-\sigma_{m, j} K_{j}^{(P)} c_{j}(t)} .
$$

Our aim here is to show that $z(t) /\left(k_{z}+z(t)\right) \prod_{j} e^{-\sigma_{m, j} K_{j}^{(P)} c_{j}(t)}>W_{0}>0$ if $t>t_{c}$ which guarantees that $\lim _{t \rightarrow \infty} x_{m}(t) / x_{m^{\prime}}(t)=0$. It follows from (C.2) that $x_{i}(t) \geq 0$ if $x_{i}(0)>0$ and thus because of (C.6) $z(t) \leq z_{0}$ and $x_{i}<\mu z_{0}$ for every $i$. Therefore, $w_{i}(t)<g_{i} z_{0} /\left(k_{z}+z_{0}\right)$ and the right hand side of (C.4) can be estimated above with

$$
\dot{c}_{j}(t)<\rho \mu \frac{z_{0}^{2}}{k_{z}+z_{0}^{2}} n_{s} g_{\max }-\left(K^{(D)} \mu z_{0} n_{s}+d\right) c_{j}(t)=\alpha_{1}-\alpha_{2} c_{j}(t)
$$

where $g_{\max }=\max \left\{g_{i}, i=1, . . n_{s}\right\}, \sum_{i=1}^{n_{s}} \eta_{i, j}$ and $\sum_{i=1}^{n_{s}} \eta_{i, j}$ can be estimated above by $n_{s}$. Here $\alpha_{1}, \alpha_{2}$ are positive constants. By introducing function $C(t)$ in such a way that its derivative estimates over $\dot{c}(t)$, we get

$$
\dot{c}_{j}(t)<\dot{C}_{j}(t)=\alpha_{1}-\alpha_{2} C(t)
$$

This estimation is valid as the ordering between derivatives guarantees $C(t)>$ $c(t)$ if $t>t^{*}$. It is easy to show that $\lim _{t \rightarrow \infty} C_{i}(t)=C^{*}$ where $C$ is a finite positive constant, thus $\lim _{t \rightarrow \infty} c_{i}(t) \leq C^{*}$ for every $i$. Similarly, knowing that $\sum_{i=1}^{n_{s}} x_{i} / \mu \leq z_{0}$ and using the estimation introduced above Eq. (C.1) can be estimated below with

$$
\dot{z}(t) \geq \dot{Z}(t)=\left(z_{0}-Z(t)\right) d-g_{\max } \frac{z_{0}}{\mu\left(k_{z}+z_{0}\right)} Z(t)
$$

Since $\lim _{t \rightarrow \infty} Z(t)=Z^{*}>0$, thus $\lim _{t \rightarrow \infty} z(t) \geq Z^{*}$. That is, $z /\left(k_{z}+\right.$ $z) \Pi_{j} e^{-\sigma_{i, j} K_{i}^{(P)} c_{j}(t)}>Z^{*} /\left(k_{z}+Z^{*}\right) \Pi_{j} e^{-\sigma_{i, j} K_{i}^{(P)} C^{*}}=W_{0}>0$ for every $t$ greater than a critical time $t_{c}$. Thus

$$
\lim _{t \rightarrow \infty} x_{m}(t) / x_{m^{\prime}}(t)=0
$$

54 as in the mixed inhibition model. We note here that the calculation remains valid if we use any monotonously decreasing function to model the effect of the antibiotic. 


\section{References}

Archetti, M., 2014. Stable heterogeneity for the production of diffusible factors in cell populations. PLOS One 9, DOI: 10.1371/journal.pone.0108526.

Arnosti, C., 2011. Microbial extracellular enzymes and the marine carbon cycle. Ann. Rev. Mar. Sci. 3, 405-425.

Bastos, M. C., Coelho, M. L., Santos, O. C., 2015. Resistance to bacteriocins produced by gram-positive bacteria. Microbiology 161, 683-700.

Bernier, S. P., Surette, M. G., 2013. Concentration-dependent activity of antibiotics in natural environments. Front. in Microbiol. 4, 20:1-14.

Borenstein, D. B., Meir, Y., Shaevitz, J. W., Wingreen, N. S., 2013. Non-local interaction via diffusible resource prevents coexistence of cooperators and cheaters in a lattice model. PLOS One 8, DOI:10.1371/journal.pone.0063304.

Cordero, O. X., Polz, M. F., 2014. Explaining microbial genomic diversity in light of evolutionary ecology. Nat. Rev. Microbiol. 12, 263-273.

Cordero, O. X., Ventouras, L., DeLong, E. F., Polz, M. F., 2012a. Public good dynamics drive evolution of iron acquisition strategies in natural bacterioplankton populations. Proc. Natl. Acad. Sci. USA 109(49), 120059-120064.

Cordero, O. X., Wildschutte, H., Kirkup, B., Proehl, S., Ngo, L., Hussain, F., Le Roux, F., Mincer, T., Polz, M. F., 2012b. Ecological populations of bacteria act as socially cohesive units of antibiotic production and resistance. Science 337, 1228-1231.

Costello, E. K., Stagaman, K., Dethlefsen, L., Bohannan, B. J. M., Relman, D., 2012. The application of ecological theory toward an understanding of the human microbiome. Science 336, 1255-1262.

Coyte, K. Z., Schluter, J., Foster, K. R., 2015. The ecology of the microbiome: Networks, competition, and stability. Science 350, 663. 
Czárán, T. L., Hoekstra, R. F., Pagie, L., 2002. Chemical warfare between microbes promotes biodiversity. Proc. Natl. Acad. Sci. USA 99, 786-790.

Dandekar, A. A., Chugani, S., Greenberg, E. P., 2012. Pleiotropy and the low cost of individual traits promote cooperation. Science 338, 264-266.

Drescher, K., Nadell, C. D., Stone, H. A., Wingreen, N. S., Bassler, B. N., 2014. Solutions to the public goods dilemma in bacterial biofilms. Curr. Biol. 24, $50-55$.

D’Souza, G., Waschina, S., Pande, S., Bohl, K., Kaleta, C., Kost, C., 2014. Less is more: Selective advantages can explain the prevalent loss of biosynthetic genes in bacteria. Evolution 68, 2559-2570, doi:10.1111/evo.12468.

Gause, G. F., 1934. The struggle for existence. Baltimore, MD: Williams \& Wilkins.

Hacker, J., Carniel, E., 2001. Ecological fitness, genomic islands and bacterial pathogenicity. EMBO Rep. 2, 376-381.

Hardin, G., 1968. The tragedy of the commons. Science 162, 1243-1248.

Hibbing, M. E., Fuqua, C., Parsek, M. R., Peterson, S. B., 2010. Bacterial competition: surviving and thriving in the microbial jungle. Nat. Rev. Microbiol. 8, 15-25 doi:10.1038/nrmicro2259.

Károlyi, G., Neufeld, Z., Scheuring, I., 2005. Rock-scissors-paper game in chaotic flow: The effect of dispersion on the cyclic competition of microorganisms. J. Theor. Biol. 236, 12-20.

Kelsic, E. D., Zhao, J., Vetsigian, K., Kishony, R., 2015. Counteraction of antibiotic production and degradation stabilizes microbial communities. Nature $521,516-519$.

Kelsic, R. D., Vetsigian, K., Kishony, R., 2016. Evolutionary stability of microbial communities with antibiotic degrading species. bioRxiv, 1-4.

URL http://dx.doi.org/10.1101/045732 
Kerr, B., Riley, M. A., Feldman, M. W., Bohannan, B. J. M., 2002. Local dispersal promotes biodiversity in real-life game of rock-paper-scissors. Nature 418, 171-174.

Koskiniemi, S., Sun, S., Berg, O. G., Anderson, D. I., 2012. Selection-driven gene loss in bacteria. Plos Genet., e1002787.

Kumar, A., Schweizer, H. P., 2005. Bacterial resistance to antibiotics: Active efflux and reduced uptake. Adv. Drug Del. Rev. 57, 1486-1513.

Kümmerli, R., T., S. K., Waldvogel, T., McNeill, K., Ackermann, M., 2014. Habitat structure and the evolution of diffusible siderophores in bacteria. Ecol. Lett. 12, 1536-1544.

Lee, M. C., Marx, C. J., 2012. Repeated, selection-driven genome reduction of accessory genes in experimental populations. Plos Genet. 8, e1002651.

Melnyk, A. H., Wong, A., Kassen, R., 2014. The fitness costs of antibiotic resistance mutations. Evol. Appl. 8, 273-283.

Miller, M. B., Bassler, B. L., 2001. Quorum sensing in bacteria. Ann. Rev. Microbiol. 55, 165-199.

Mitri, S., Foster, K. R., 2016. Pleiotropy and the low cost of individual traits promote cooperation. Evolution 70, 488-494, DOI: 10.1111/evo.12851.

Morris, J. J., 2015. Black queen evolution: the role of leakiness in structuring microbial communities. Trends in Genetics 31, 475-482.

Morris, J. J., Lenski, R. E., Zinser, E. R., 2012. The black queen hypothesis: evolution of dependencies through adaptive gene loss. mBio e00036-12.

Morris, J. J., Papoulis, S. e., Lenski, R. E., 2014. Coexistence of evolving bacteria by shared black queen function. Evolution 68, 2960-2971.

Müller, A. P. O., Gallas, J. A. C., 2010. How community size affects survival chances in cyclic competition games that microorganisms play. Phys. Rev. E $82,052901$. 
Ross-Gillespie, A., Gardner, A., Buckling, A., West, S. A., Griffin, A. S., 2009.

Density dependence and cooperation: theory and a test with bacteria. Evolution $63,2315-2325$.

Ross-Gillespie, A., Gardner, A., West, S. A., Griffin, A. S., 2007. Frequency dependence and cooperation: theory and a test with bacteria. Am. Nat. 170, $331-342$.

Scheuring, I., 2014. Diffusive public goods and coexistence of cooperators and cheaters on a 1d lattice. PLOS One 9, DOI:10.1371/journal.pone.0100769.

Velend, M., 2010. Conceptual synthesis in community ecology. Quart. Rev. Biol. 85, 183-206.

Weibull, J. W., 1997. Evolutionary Game Theory. The MIT Press Cambridge, Massachusets, London, England.

West, S. A., Griffin, A. S., A., G., Diggle, S. P., 2001. Social evolution theory for microorganisms. Nat. Rew. Microbiol. 4, 597-607.

Wright, G. D., 2005. Bacterial resistance to antibiotics: enzymatic degradation and modification. Adv. Drug Deliv. Rev. 57, 1451-1470. 

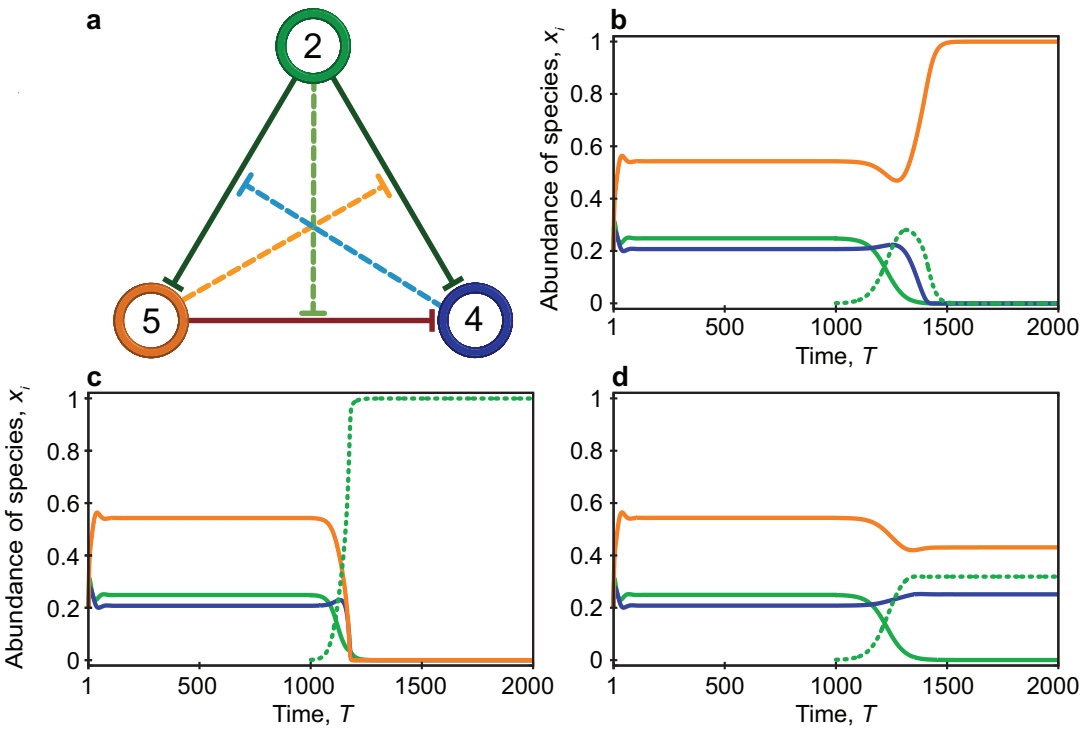

Figure 3: Invasion dynamics of different production cheaters in a model community. (a) The interaction topology of the model community. Each species produces different antibiotics, and species numbering represents the increments in reproduction rates as described in Methods. Species 2 is not affected by any antibiotic, species 5 is inhibited by antibiotic produced by species 2 , and species 4 is inhibited by two different antibiotics produced by species 2 and 5 . Three different scenarios of production cheater mutant (depicted by dashed lines) invasions: (b) both the introduced mutant and the corresponding mother species go extinct after the invasion of production cheater mutant for species 2 (that ceases producing the antibiotic that inhibits species 5 , depicted by the green dashed line), (c) the invasion of production cheater mutant of species 2 (that ceases producing the antibiotic that inhibits species 4 , depicted by the green dashed line) results in the exclusion of the mother type and triggers further species loss, and finally (d) the production cheater mutant of species 2 (that ceases producing the antibiotic that inhibits species 4 , depicted by the green dashed line), similarly as in the previous numerical experiment, but with lower fitness advantage, replaces the mother lineage. Parameters are the same as in Fig. $2, \alpha=0.05$ for $(\mathbf{b}, \mathbf{d}), \alpha=0.1$ for $(\mathbf{c})$. Orange, green, blue solid lines correspond to species 5, 2, 4, respectively. Dashed line denotes the actual mutant colored similarly as its mother species. 

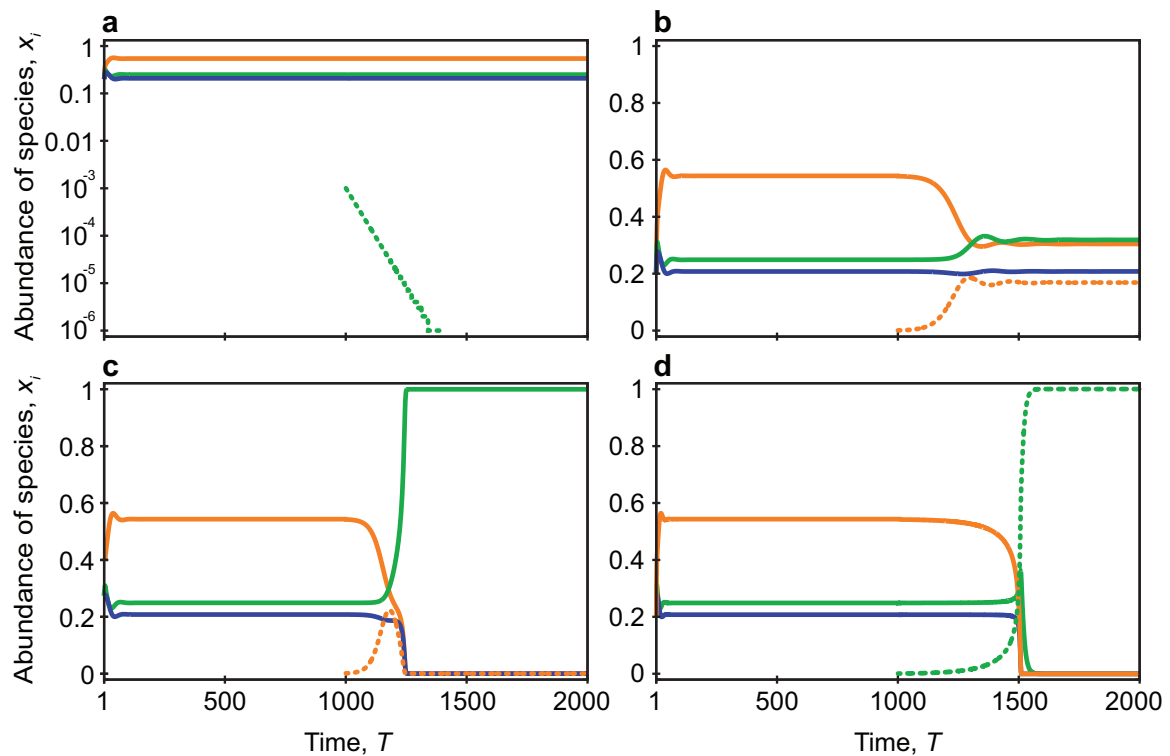

Figure 4: Four different scenarios for the invasion of degradation cheater mutants (dashed lines) in model communities depicted by Figure 3a. (a) Unsuccessful invasion of the degradation mutant of species 2 (that ceases to produce the factor degrading the antibiotic produced by species 5 , depicted by the green dashed line), where the resident community remains unchanged after the invasion attempt. (b) Successful invasion of degradation mutant of species 5 (that ceases to produce the factor degrading one of the antibiotics produced by species 2 , depicted by the orange dashed line), leading to the coexistence of all species, the residents and the mutant. (c) The invasion of degradation mutant of species 5 fails, but triggers species extinctions in the community, and one resident species survives in the end. (d) The mutant of species 2 successfully invades a stable community and excludes all other species. Parameters and color coding are the same as in Figure 3, $\alpha=0.05$ for $\mathbf{a}$ and $\mathbf{b}, \alpha=0.08$ for $\mathbf{c}$, and $\alpha=0.1$ for $\mathbf{d}$. 